\title{
Utilização de coalescedor com leito de resinas de trocas catiônicas para pré-tratamento de óleo emulsionado em efluentes líquidos
}

\author{
Use of coalescer with a bed made of cationic exchange resins \\ for pre-treatment of emulsified oil from oily wastewater
}

\begin{abstract}
Albérico Ricardo Passos Motta', Cristiano Piacsek Borges², Asher Kiperstok ${ }^{3}$, Karla Patricia Esquerre ${ }^{4}$
\end{abstract}

\begin{abstract}
RESUMO
O tratamento de efluentes com coalescedor de leito consiste em induzir o aumento das gotas de óleo, pela passagem da emulsão por um leito poroso, para que elas possam, posteriormente, flotar por processo de separação gravitacional. O objetivo deste artigo foi apresentar considerações técnicas e experimentais sobre o uso do coalescedor de leito granular, com leito constituído por resinas de trocas catiônicas, visando o pré-tratamento de óleo emulsionado em efluentes líquidos. Os testes ocorreram em uma unidade em escala de bancada alimentada com emulsão óleo em água (O/A) com um teor de óleos e graxas (TOG) entre 200 e 400 mg.L.-1 e diâmetro de gotas entre 3 a $8 \mu \mathrm{m}$. As velocidades do fluido ( $v$ ) testadas foram entre $4,4 \mathrm{e} 17,4 \mathrm{~m} \cdot \mathrm{h}^{-1}$. A altura do leito do coalescedor foi fixada em $5 \mathrm{~cm}$. Os parâmetros usados para avaliar a eficiência dos testes foram eficiência de remoção de óleo (\%E), pressão diferencial através do leito $(\Delta \mathrm{p})$ e permeabilidade do leito (ko). Os resultados mostraram que o processo operou em estado estacionário e que os valores de TOG testados influenciaram no processo, bem como as velocidades do fluido, cuja elevação reduziu a eficiência significativamente. Os valores aproximados de \%E obtidos foram 50 e 20-30\%, para valores de $\checkmark$ de 4,4 e $17,4 \mathrm{~m}^{-1} \mathrm{~h}^{-1}$, respectivamente.
\end{abstract}

Palavras-chave: óleo; emulsão; coalescência; leito poroso.

\begin{abstract}
The wastewater treatment with a bed coalescer consists of inducing the increase of oil droplets by passing the emulsion through a porous bed, so they can flote by gravitational separation process. The objective of this paper is to present technical and experimental considerations on the use of a granular bed coalescer, with a bed made of cationic exchange resins, for pre-treatment of emulsified oil from oily wastewater. Tests were conducted on a bench scale unit, which was fed with oil in water emulsion with oil and grease concentration (O\&G) between 200 and 400 mg. $\mathrm{L}^{-1}$ and oil droplet diameter between 3 and $8 \mu \mathrm{m}$. The fluid velocities ( $\mathrm{v}$ ) tested were between 4.4 and $17.4 \mathrm{~m} \cdot \mathrm{h}^{-1}$. The coalescer bed height was fixed at $5 \mathrm{~cm}$. The parameters used for assessing the tests efficiency were oil removal efficiency percentage (\%E), differential pressure across the bed and, bed permeability. Results showed that the process operates in a steady-state condition and that the influent $O \& G$ affected the process efficiency, as well as the fluid velocities which reduced the efficiency significantly, with its increasing. The \%E values obtained were between 50 and 20-30\% to $v$ between 4.4 and 17.4 m.h.'- respectively.
\end{abstract}

Keywords: oil; emulsion; coalescence; porous bed.

\footnotetext{
${ }^{1}$ Engenheiro Civil. Engenheiro Sanitarista pela Universidade Federal da Bahia (UFBA). Mestre em Engenharia Ambiental pela Newcastle University, Newcastle upon Tyne. Doutor em Engenharia Industrial pela UFBA. Engenheiro de Meio Ambiente da Petrobras - Salvador (BA), Brasil.

²Engenheiro Químico pela Universidade Federal do Rio de Janeiro (UFRJ). Mestre e Doutor em Engenharia Química pela UFRJ. Professor do Programa de Engenharia Química do Instituto Alberto Luiz Coimbra da UFRJ - Rio de Janeiro (RJ), Brasil.

${ }^{3}$ Engenheiro Civil pelo Instituto Tecnológico de Israel (TECHNION). Mestre e Doutor em Engenharia Química/Tecnologias Ambientais pela University of Manchester. Coordenador do TECLIM - Rede de Tecnologias Limpas. Professor do Programa de Engenharia Industrial da Escola Politécnica da UFBA - Salvador (BA), Brasil. ${ }^{4}$ Engenheira Química pela Universidade Federal de Alagoas (UFAL). Mestre e Doutora em Engenharia Química pela Universidade Estadual de Campinas (UNICAMP). PósDoutora em Engenharia Socioambiental pela Hokkaido University. Professora do Departamento de Engenharia Química e do Programa de Pós-Graduação em Engenharia Industrial da Escola Politécnica da UFBA - Salvador (BA), Brasil.

Endereço para correspondência: Albérico Ricardo Passos Motta - Avenida Tancredo Neves, 1.368, 80 andar, Sala 802 - Caminho das Árvores - 41820-O21 - Salvador (BA), Brasil - E-mail: arpmotta@yahoo.com.br

Recebido: 06/05/13 - Aceito: 14/10/14 - Reg. ABES: 116813
} 


\section{INTRODUÇÃO}

Os separadores gravitacionais, largamente utilizados nas instalações industriais, além de remover óleo livre (gotas de óleo acima de $100 \mu \mathrm{m}$ ), promovem uma primeira separação de óleo com gotas maiores do que $10 \mu \mathrm{m}$. Apesar disso, essas unidades não são suficientemente efetivas para as gotas remanescentes de menores diâmetros, que flotam muito mais lentamente (SPIELMAN, 1968), aspecto que implicaria em unidades com tempo de detenção elevado a ponto de as tornarem inviáveis (AUSTIN, 1979).

Um aspecto agravante é que, em grande parte dos casos, as gotas de óleo de pequenas dimensões (abaixo de $10 \mu \mathrm{m}$ ) podem se constituir em uma fração muito pequena do volume da dispersão, o que a torna demasiadamente diluída para que as gotas se choquem entre si e que, assim, ocorra a coalescência - junção de duas ou mais gotas de óleo, formando uma gota maior - de forma espontânea. Ademais, algumas dispersões contêm tensoativos naturais, compostos que podem inibir a coalescência (THOMAS, 2001). Esse é o caso da água produzida de petróleo, em que agentes tensoativos solúveis em água e finos sólidos minerais são adsorvidos na superfície das gotas, dificultando, assim, o processo de desemulsificação (LI \& GU, 2005).

O processo de tratamento com coalescedor de leito consiste em induzir a separação das gotas de óleo emulsionadas em água pela passagem da emulsão através de um leito poroso. Nesse caso, as gotas pequenas são retidas no leito até que ocorra o seu crescimento por coalescência. As gotas maiores, então, são arrastadas para fora do material do leito pelas forças dinâmicas, uma vez que, agora, já possuem um tamanho tal que faz com que elas possam flotar por separação gravitacional (AUSTIN, 1979).

Os dois tipos de leito usualmente utilizados são o leito de fibras e o granular. O leito de fibras pode ser constituído por materiais diversos empacotados e compactados, como fibra de vidro ou polímeros diversos, enquanto o leito granular pode ser constituído por materiais como vidro, plástico ou pedras, com dimensões diversas. Ambos são capazes de forçar a separação do óleo por coalescência, alcançando uma boa eficiência. Entretanto, eles são muito susceptíveis a problemas de bloqueio de poros devido à presença de sólidos em suspensão.

Uma das vantagens do leito de fibras é a sua flexibilidade; como algumas fibras são compressíveis, a permeabilidade do leito (ko) pode ser alterada de modo que uma grande variedade de valores desse parâmetro possa ser estudada para uma mesma altura de leito. Outra vantagem é que o leito de fibras pode ser confeccionado para ter porosidade e superfície específica maiores do que os leitos granulares grosseiros e, com isso, obter uma melhor separação de fases. Isso ocorre porque quanto maior a superfície específica, maior o número de colisões das gotas com o material do leito, elevando, assim, a eficiência do processo (SOKOLOVIĆ et al., 2003).
Apesar dessas vantagens, o leito de fibras é mais vulnerável a obstruções por sólidos, que, às vezes, são inevitáveis. Outra desvantagem é que a sua estrutura dificulta sobremaneira o processo de limpeza (LI \& GU, 2005), fato que não ocorre com leito granular, que pode ser facilmente lavado em caso de obstrução dos poros.

O coalescedor de leito opera de forma similar a um filtro rápido de uma estação de tratamento de água. Alguns dos mecanismos de captura das partículas (impacto inercial, interceptação indireta, difusão e atração eletrostática) são similares, nos dois processos, inclusive. Entretanto, existem diferenças fundamentais entre ambos: o coalescedor opera em estado estacionário (se não houver presença de sólidos suspensos na emulsão) e a concentração de material que entra no leito é igual à concentração de saída do mesmo. A importância do leito está no fato de que ele provoca um aumento do diâmetro das gotas de óleo na saída. Com diâmetros maiores, as gotas flotam com uma velocidade muito maior (SHERONY \& KINTNER, 1971).

Vários fatores afetam a eficiência do processo de coalescência e separação, como propriedades do leito, propriedades dos grãos, velocidade do fluido (v), propriedades de ambos os líquidos, fenômenos superficiais, a configuração hidráulica da unidade e a interdependência de algumas variáveis entre si (SOKOLOVIĆ; VULIĆ; SOKOLOVIĆ, 2007).

Entre esses fatores, a v desempenha um importante papel, uma vez que influencia vários dos mecanismos de captura das gotas. Alguns autores (SAREEN et al., 1966; SPIELMAN, 1968) usaram o termo velocidade crítica $\left(\mathrm{v}_{\mathrm{c}}\right)$, definida como a velocidade a partir da qual o teor de óleos e graxas (TOG) na alimentação do coalescedor (TOGac) influencia, de modo significativo, no TOG de saída do coalescedor (TOGsc); abaixo da $\mathrm{v}_{\mathrm{c}}$ essa influência ocorre apenas de forma bastante reduzida.

$A v_{c}$ foi usada por alguns pesquisadores como referência para avaliar os efeitos de determinados parâmetros na eficiência do processo. Trabalhando com leito de fibras de poliuretano, com TOGac com valores entre 500 e 2.000 mg. $\mathrm{L}^{-1}$, diâmetro médio de gotas do óleo no efluente testado (Øg) de $20 \mu \mathrm{m}$, v entre 10 e $45 \mathrm{~m} . \mathrm{h}^{-1}$ e altura do leito com valores entre 3 e 15 cm, Sokolović, Sokolović e Doković (1997) verificaram que os efeitos do TOGac e da altura do leito na eficiência do processo são desprezíveis para velocidades menores do que $\mathrm{a}_{\mathrm{c}}$. Para valores de $\mathrm{v}$ acima de $\mathrm{v}_{\mathrm{c}}$, no entanto, a influência do TOGac e da altura do leito na eficiência do processo muda; no caso do TOGac, quanto maior o seu valor, maior o TOGsc, enquanto no caso da altura do leito verificou-se que existe um valor intermediário desse parâmetro que pode ser considerado ótimo, pois é onde ocorre a eficiência máxima.

O processo de coalescência por leitos granulares já é conhecido desde o começo do século passado, sendo que suas aplicações em grande escala surgiram na década de 1930 (SHERONY \& KINTNER, 1971). Entretanto, apesar do fato de que nas últimas décadas as pesquisas nessa área foram intensificadas consideravelmente, o processo de coalescedor de leito é um fenômeno complexo e afetado por um grande número de 
variáveis. Dessa forma, o projeto de um coalescedor depende da realização de testes em unidades de bancada (GOVEDARICA et al., 2013).

O objetivo deste artigo foi apresentar considerações técnicas e experimentais sobre o tratamento de efluentes oleosos por meio de um coalescedor com leito constituído por resinas de trocas catiônicas, visando o pré-tratamento dos efluentes, com base em testes realizados em uma unidade experimental de bancada.

\section{MATERIAIS E MÉTODOS}

A seguir são descritos os detalhes do efluente sintético gerado, da unidade experimental de bancada, da limpeza química do coalescedor, das análises de laboratório dos parâmetros testados e investigados e as condições dos experimentos.

\section{O efluente gerado}

O efluente utilizado nos testes foi produzido pela unidade geradora de emulsões (UGE), uma planta industrial que gera amostras de emulsão óleo em água (O/A) sintética. Essa planta fica instalada no Laboratório de Tratamento e Reuso de Águas (LARA) do Centro de Pesquisas e Desenvolvimento Leopoldo Américo Miguez de Mello (Cenpes/Petrobras). A UGE tem condições de produzir efluentes com diferentes TOG e com diferentes diâmetros médios de gotas de óleo $(\emptyset \mathrm{g})$, podendo-se alcançar até valores na faixa de 1 a $2 \mu \mathrm{m}$.

O óleo utilizado no preparo dessa emulsão foi petróleo leve, proveniente do campo de Roncador, no Rio de Janeiro. Suas principais características são: viscosidade cinemática a $50^{\circ} \mathrm{C}$ de $9,950 \mathrm{~mm}^{2} . \mathrm{s}^{-1}$, densidade API de $28,3^{\circ}$ e densidade relativa de $0,8824 \mathrm{~kg} . \mathrm{L}^{-1}$. A densidade baixa é um aspecto de grande importância para os testes realizados, pois facilita sobremaneira o manuseio e preparo do efluente oleoso a ser usado como amostra.

\section{A unidade experimental}

Os recursos de aquisição de componentes e de montagem da unidade experimental de bancada (UExp) utilizada neste estudo foram originários do Laboratório de Membranas da Coppe (UFRJ). A unidade ficou instalada no LARA (Cenpes), onde, ao longo do seu tempo de utilização, passou por uma série de serviços de manutenção e otimização.

A UExp está apresentada na Figura 1. O coalescedor experimental utilizado foi constituído por um tubo de PVC, disposto na posição vertical, com diâmetro de $100 \mathrm{~mm}$ e altura de $85 \mathrm{~cm}$. O tubo continha, no seu interior, o leito granular objeto deste estudo e mais alguns dispositivos internos que tinham a função de dar suporte a esse leito e de atenuar a turbulência. Para controle operacional foram medidas

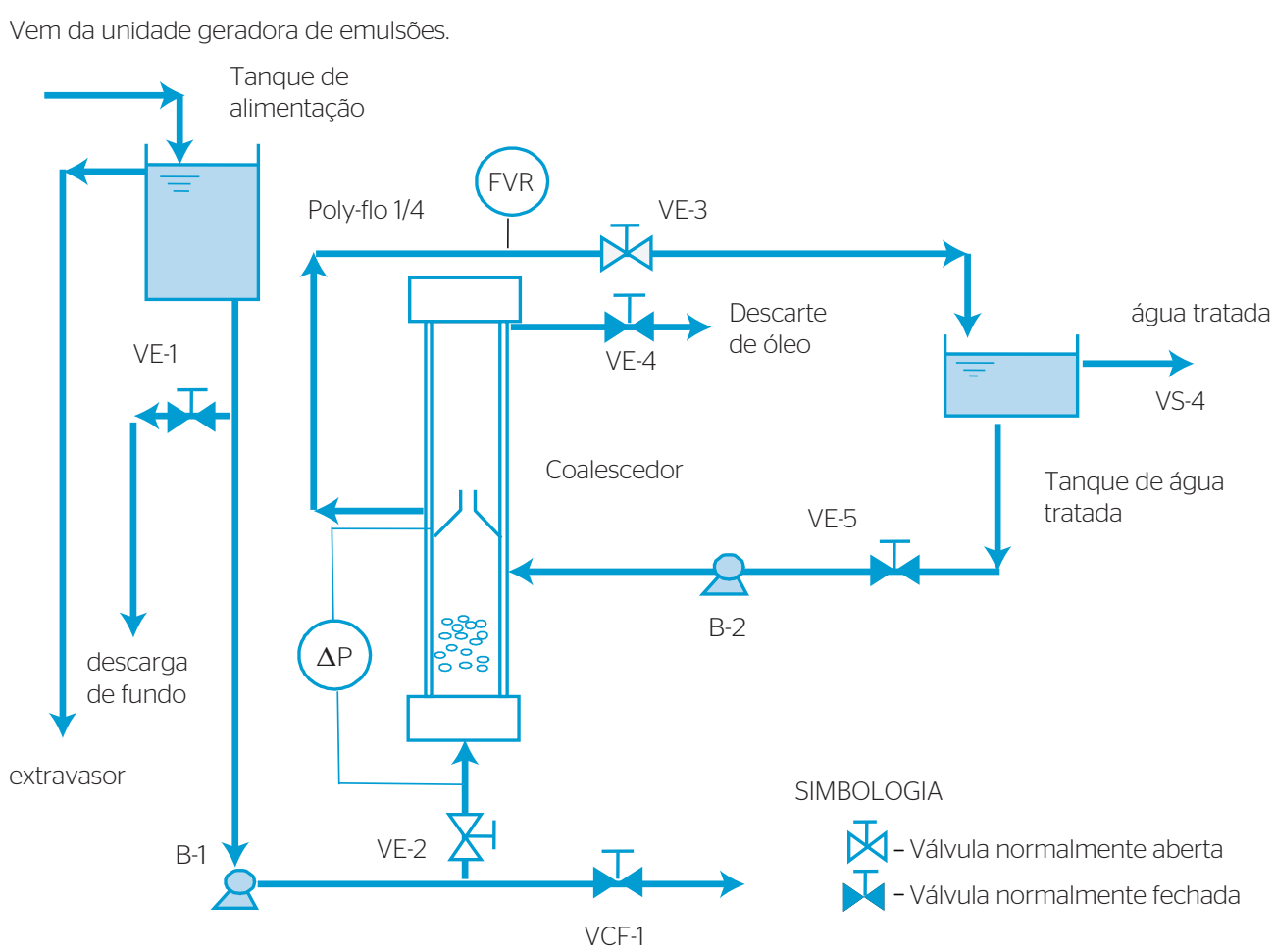

FVR: Medidor de vazão; VCF: válvula controladora de vazão; $\Delta$ p: manômetro diferencial; VE: válvula on/off; B-1 e B-2: bombas hidráulicas

Figura 1 - Fluxograma da unidade experimental para o pré-tratamento de efluentes oleosos por coalescedor de leito granular. 
a vazão de saída do coalescedor (Qsc) e a pressão diferencial através do leito $(\Delta \mathrm{p})$.

O leito granular possuía uma porosidade média de 0,40 e era formado por resinas de trocas cationnicas, do fabricante Rohm and Haas/ Dow Química, com diâmetros entre 0,425 e 1,275 mm.

\section{Limpeza do leito do coalescedor}

Foram realizados dois tipos de testes em termos de tempo de duração, um de curta e o outro de longa duração. Para os primeiros, que ocorreram ao longo de 7 horas, foram realizados 14 testes. Após o final de cada teste, a limpeza do leito era realizada para que os testes seguintes pudessem ser efetuados. A limpeza foi feita com tensoativos iônicos. Os testes de longa duração ocorreram em dias consecutivos, ou seja, o teste era interrompido no final de cada dia e reiniciado no dia seguinte, tomando-se o cuidado de que a interrupção não causasse modificações significativas no leito. Nesse caso, a lavagem do leito ocorria apenas no final do último dia de teste. Foram realizados dois testes de longa duração.

O objetivo da limpeza foi recuperar a ko para permitir a realização de um novo teste. Após a limpeza, eram feitas novas medições de permeabilidade, realizadas com água proveniente da rede pública de água potável. O valor da permeabilidade média do leito encontrado foi de $8,84 \times 10^{-5} \pm 1,0029 \times 10^{-5}$.

O produto químico utilizado foi detergente neutro, em solução aquosa, e o procedimento utilizado foi dividido em três etapas. Na primeira, a solução era percolada através do leito e descartada continuamente. Na segunda etapa, uma nova solução com detergente era recirculada através do leito e, apenas após cerca de uma hora, era descartada. A última etapa consistia em veicular água através do leito, mantendo o descarte contínuo por cerca de uma hora. Se, após a limpeza, o valor da permeabilidade medida estivesse abaixo da faixa adequada, a limpeza era refeita.

\section{Análises de laboratório e coleta de dados}

As determinações de TOG foram feitas por intermédio da sua correlação com as medidas da turbidez, utilizando o turbidímetro portátil Hach 2100P. O objetivo do uso da turbidez foi a obtenção de um método simplificado e alternativo, em substituição aos usuais métodos de medição de TOG, feitos por meio da absorbância e que utilizam extração prévia do óleo da emulsão pela adição de um solvente, sendo, portanto, extremamente trabalhosos e consumidores de tempo.

Para tanto, foram preparadas várias amostras de óleo com concentrações variando entre 50 e 800 mg.L $L^{-1}$ e, em seguida, medidos os valores da turbidez dessas amostras. Os resultados obtidos foram representados em uma curva que resultou em uma relação linear entre turbidez e TOG com um coeficiente de correlação de 0,92 , considerado bom.
Para determinação do diâmetro de gotas, o equipamento utilizado foi o MALVERN MASTER SIZER, fabricado pela Malvern Instruments. O MALVERN tem capacidade de determinação de diâmetro médio de partículas de 0,02 até $2.000 \mu \mathrm{m}$.

\section{Condições dos experimentos}

As condições operacionais testadas foram: TOGac com valores de 200 a 400 mg.L $L^{-1}$, $\emptyset$ g entre 3 a $8 \mu \mathrm{m}$ e vazão de alimentação do coalescedor (Qac) com valores entre 30 e 120 L.h ${ }^{-1}$. Essas vazões equivalem a valores de v entre 4,4 e 17,4 m.h. ${ }^{-1}$. A altura do leito do coalescedor foi fixada em $5 \mathrm{~cm}$, valor utilizado com base nos usualmente encontrados na literatura para tal processo (GOVEDARICA et al., 2013; SOKOLOVIĆ; SOKOLOVIĆ; DOKOVIĆ, 1997) e considerando-se que o objetivo era de pré-tratamento, em vez de tratamento.

Os parâmetros utilizados para avaliar a eficiência do processo em função das condições operacionais testadas foram TOGsc, eficiência de remoção de óleo (\%E), $\Delta \mathrm{p}$ e ko.

Sobre a permeabilidade, foram estudados vários aspectos ligados ao seu comportamento a fim de se verificar se o sistema operou em estado estacionário ou dinâmico. Para tanto, foram realizados ensaios de longa duração com valores de TOGac de 200 e 400 mg.L.-1 e valor de $\mathrm{v}$ de $4,4 \mathrm{~m} \cdot \mathrm{h}^{-1}$. O valor da permeabilidade foi calculado por meio da lei de Darcy para meios porosos com fluxo ascendente.

Além disso, foram avaliadas as influências de dois outros importantes parâmetros na eficiência do processo: TOGsc e v. A avaliação da influência do TOGac foi realizada para os seus valores com $200 \mathrm{e}$ $400 \mathrm{mg} . \mathrm{L}^{-1} \mathrm{e} \mathrm{v}$ com valores de 4,4 e 17,4 m.h-1 ${ }^{-1}$, enquanto para a influência da velocidade foram testados os valores de 4,4, 8,7 e 17,4 m.h.1

\section{RESULTADOS E DISCUSSÃO}

\section{Verificação da permeabilidade}

Uma das etapas iniciais do estudo consistiu em verificar o comportamento do processo, em termos do que ocorre no interior do leito do coalescedor. Para tanto, foram avaliados dois parâmetros, em um teste de longa duração: $\Delta \mathrm{p}$ e ko. A permeabilidade adotada foi a normalizada, expressa por ko/ko-inicial, sendo ko-inicial a permeabilidade no tempo inicial.

A Figura 2 apresenta a evolução de $\Delta \mathrm{p}$ e ko/ko-inicial ao longo do tempo para $\mathrm{v}$ de $4,4 \mathrm{~m} \cdot \mathrm{s}^{-1} \mathrm{e}$ dois valores diferentes de TOGac, 200 e $400 \mathrm{mg} \cdot \mathrm{L}^{-1}$.

Conforme pode ser observado, ocorre uma queda da permeabilidade e esse processo é verificado por dois estágios bastante distintos. No primeiro, ocorre uma queda bastante acentuada, processo que transcorre do início da operação a até poucas horas após o seu início (em torno de três a quatro horas, nesse caso). O segundo estágio se 
caracteriza pela manutenção da permeabilidade com valor constante, ao longo do tempo, formando um patamar praticamente horizontal. Essa situação perdurou até o final do teste, decorridas entre 19 ou 20 horas de operação.

De fato, a queda da permeabilidade em dois estágios distintos é um comportamento típico do processo de coalescência em leito granular. Esse aspecto pode ser melhor entendido quando são avaliadas as possibilidades de captura das gotas, descritas a seguir. A captura de uma gota suspensa em uma fase fluida contínua é possível devido à colisão dessa gota conforme uma ou mais das três possibilidades seguintes: com outra gota suspensa na dispersão, com os grãos do leito ou com outras gotas já capturadas e aderidas ao leito (SAREEN et al., 1966). Dessas três possibilidades, a terceira é a que mais influencia no processo de captura das gotas. Em outras palavras, a taxa de captura das gotas se eleva consideravelmente quando já existem gotas aderidas ou mantidas dentro dos interstícios do leito e fixadas à sua estrutura. Por outro lado, a probabilidade de captura por colisão das gotas da fase suspensa entre si mesmas é muito baixa (SAREEN et al., 1966).

Neste estudo, como era feita a limpeza do leito após o fim de cada teste, os seus grãos ainda estavam limpos, no primeiro estágio, e com poucas gotas de óleo a eles fixadas. Nesse caso, no período inicial do teste, a captura das gotas ocorria, predominantemente, devido à colisão das mesmas com a obstrução da estrutura do leito, formada, nesse caso, pelos grãos.

Dessa forma, no segundo estágio, a captura das gotas ocorre, principalmente, devido à colisão das mesmas com outras gotas que foram capturadas pelo leito e estão ligadas à sua estrutura.

Sendo assim, no estágio inicial, a permeabilidade é elevada, pois o leito ainda está limpo e com sua porosidade original. À medida que as gotas de óleo começam a se fixar nos grãos, a porosidade aparente é reduzida e, consequentemente, a permeabilidade cai.

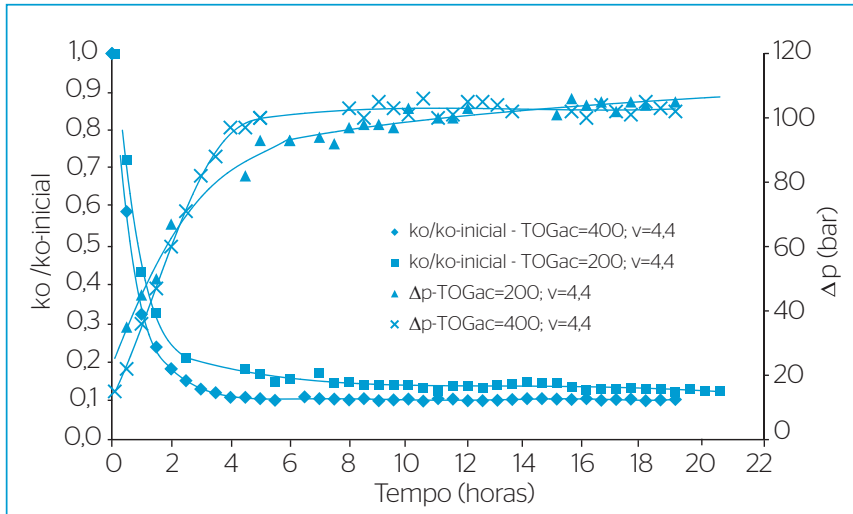

$\Delta$ p: pressão diferencial através do leito; TOGac: teor de óleos e graxas na alimentação do coalescedor; ko/ko-incial: permeabilidade do leito normalizada.

Figura 2 - Evolução da pressão diferencial e da permeabilidade do leito do coalescedor ao longo do tempo para teor de óleos e graxas na alimentação do coalescedor de 200 e $400 \mathrm{mg.L}^{-1}$ e velocidade do fluido de 4,4 m.s. ${ }^{-1}$ (Qac=30 L.h.' $)$.
Destaca-se que os coalescedores de leito podem operar tanto em estado dinâmico quanto em estado estacionário. No dinâmico, a pressão diferencial entre a alimentação e a saída do leito aumenta continuamente com o tempo, enquanto no estacionário ela é constante (SPIELMAN \& SU, 1977). Neste estudo, o segundo estágio da figura mostra que o coalescedor opera em estado estacionário e que, nas amostras testadas, a concentração de sólidos suspensos ou outro material particulado é relativamente baixa a ponto de não influenciar no processo de forma significativa.

\section{Influência do teor de óleos e graxas de alimentação na eficiência}

O TOGacé um dos parâmetros que podem afetar a eficiência do processo. Neste estudo, para $\mathrm{v}=4,4 \mathrm{~m} \cdot \mathrm{h}^{-1}$ foram realizados 6 e 5 testes, para valores de TOGac de 200 e $400 \mathrm{mg} . \mathrm{L}^{-1}$, respectivamente. Para v=17,4 m.h $\mathrm{h}^{-1}$ foi realizado apenas um teste para cada valor de TOGac. Para essas condições testadas verificou-se que os valores de TOGac influenciaram na eficiência de remoção de óleo.

A Figura 3 mostra o comportamento da eficiência de remoção de óleo ao longo do tempo para os valores de TOGac de 200 e 400 mg.L $\mathrm{L}^{-1}$. Para os testes realizados com os mesmos parâmetros mais de uma vez são apresentados os valores das médias e os respectivos desvios padrão por ponto.

Um aspecto relevante observado no gráfico foi que os maiores valores de eficiência são encontrados para os maiores valores de TOGac. Isso se verifica tanto para a velocidade de 4,4 quanto para a de $17,4 \mathrm{~m} \cdot \mathrm{s}^{-1}$, principalmente para esta última, em que essa diferença de eficiência fica ainda mais evidente. É provável que isso se deva ao fato de que uma maior concentração de óleo resulte em uma maior colisão das gotas entre si, independentemente do leito. Esse aspecto pode ser relevante

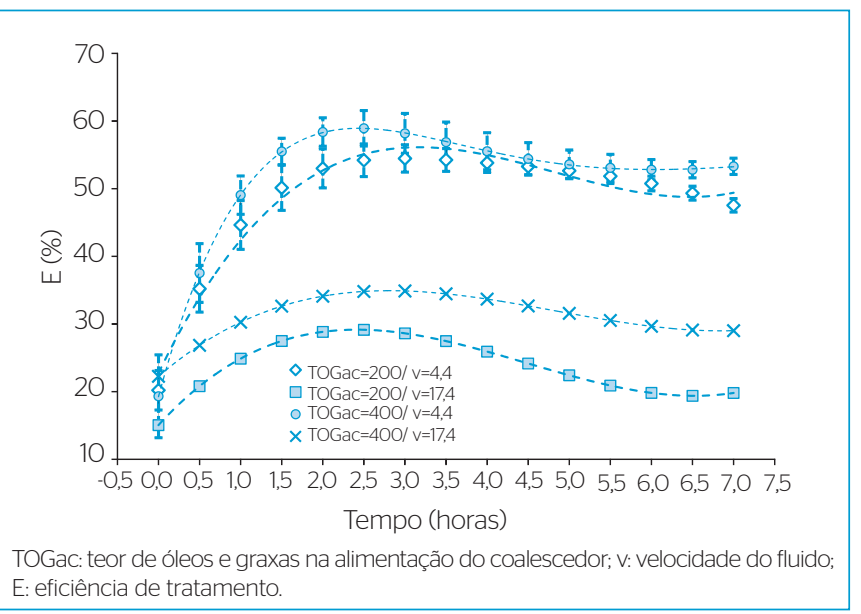

Figura 3 - Influência do valor de teor de óleos e graxas na alimentação do coalescedor (200 e $400 \mathrm{mg}^{\mathrm{L}^{-1}}$ ) na eficiência de tratamento ao longo do tempo, para valores de velocidade do fluido de 4,4 e $17,4 \mathrm{~m} \cdot \mathrm{h}^{-1}$. 
(LI \& GU, 2005), apesar ter sido citado que a probabilidade de ocorrência da captura por colisão das gotas da fase suspensa entre si mesmas é muito baixa (SAREEN et al., 1966).

Li e Gu (2005) acrescentaram que, com o aumento do TOGac, a densidade de gotículas de óleo no interior da emulsão aumenta, considerando-se que a distribuição de tamanho de gotículas de óleo permaneça inalterada. Consequentemente, o aumento da densidade de gotas de óleo resulta em um aumento de colisões entre elas, o que pode melhorar a eficiência global de coalescência.

Outro aspecto relevante foi que a intensidade da citada influência do TOGac na eficiência depende da v. Observa-se que, para a maior das duas velocidades $\left(17,4 \mathrm{~m} \cdot \mathrm{s}^{-1}\right)$, a diferença entre os valores das eficiências de cada um dos dois TOGac testados foi maior, aspecto que demonstra, com clareza, que existe uma interação entre os parâmetros TOGac e v.

Ressalta-se que já se verifica aqui a influência da velocidade na eficiência do processo. Dos dois valores de velocidade considerados, a eficiência foi menor para o maior dos dois valores $\left(17,4 \mathrm{~m} \cdot \mathrm{h}^{-1}\right)$. A influência da velocidade será vista a seguir.

\section{Influência da velocidade do fluido na eficiência}

A v representa um parâmetro de extrema importância para o correto desempenho do processo, pois é responsável pelo controle de vários outros fenômenos, como o mecanismo e a probabilidade de captura das gotas de óleo, a distribuição da fase dispersa nos regimes de fluxo de óleo e a redispersão do óleo retido (SOKOLOVIĆ; GOVEDARICA; SOKOLOVIĆ, 2010).

As Figuras 4A e 4B mostram o comportamento da eficiência de remoção de óleo ao longo do tempo para valores de TOGac de 200 e $400 \mathrm{mg} \cdot \mathrm{L}^{-1}$ e diferentes valores de velocidade: $4,4,8,7$ e $17,4 \mathrm{~m} \cdot \mathrm{h}^{-1}$. Foram realizados três testes para $\mathrm{v}=8,7 \mathrm{~m} \cdot \mathrm{h}^{-1}\left(\mathrm{TOGac}=200 \mathrm{mg} \cdot \mathrm{L}^{-1}\right)$.
Para os testes realizados com os mesmos parâmetros mais de uma vez são apresentados os valores das médias e dos respectivos desvios padrão por ponto.

Pode-se observar, pelo gráfico, que a eficiência decresce à medida que a velocidade aumenta. A maior eficiência do processo é obtida para o menor valor de velocidade testado $\left(4,4 \mathrm{~m} \cdot \mathrm{h}^{-1}\right)$, enquanto a menor eficiência é obtida para o maior valor de v, ou seja, 17,4 m.h-1. Esse aspecto é esperado, uma vez que a velocidade influencia nos mecanismos de captura das partículas (impacto inercial, interceptação indireta, difusão e atração eletrostática).

Para identificação da $v_{c}$ foi traçada uma curva da variação dos valores de TOGsc em função da velocidade para o TOGac de $200 \mathrm{mg} \cdot \mathrm{L}^{-1}$ decorridas 4 horas após iniciados os testes (Figura 5). Foram realizados três testes para $\mathrm{v}=5,8 \mathrm{~m} \cdot \mathrm{h}^{-1}$.

Como pode ser observado, para valores de v acima de 4,4 m.h.1 , há uma variação do TOGsc linearmente proporcional em relação à variação da velocidade. Essa variação é significativa, uma vez que resulta em um acréscimo considerável do TOGsc para um aumento de v.

Neste trabalho, conforme visto na Figura 3, o TOGac influenciou no TOGsc e na \%E, tanto para o valor mais baixo de $\mathrm{v}$ testado $(4,4)$ quanto para o mais elevado $(17,4)$. Para esta última, essa influência foi ainda mais acentuada.

Esses aspectos citados dão um indicativo de que as velocidades testadas estão acima de $\mathrm{v}_{\mathrm{c}}$ (Figura 5). Além disso, como a influência do TOGac na eficiência foi relativamente pequena para a velocidades de $4,4 \mathrm{~m} \cdot \mathrm{h}^{-1}$, pode-se inferir que $\mathrm{a}_{\mathrm{c}}$ está próxima desse valor.

Destaca-se o fato de que os valores de TOGac utilizados neste trabalho (200 e 400 mg.L $\mathrm{L}^{-1}$ ) estão fora da faixa testada por Sokolović, Sokolović e Doković (1997): entre 500 e 2.000 mg.L $L^{-1}$. Ainda assim, os resultados experimentais ligados a influência de v no TOGsc, para $\mathrm{v}>\mathrm{v}_{\mathrm{c}}$
A

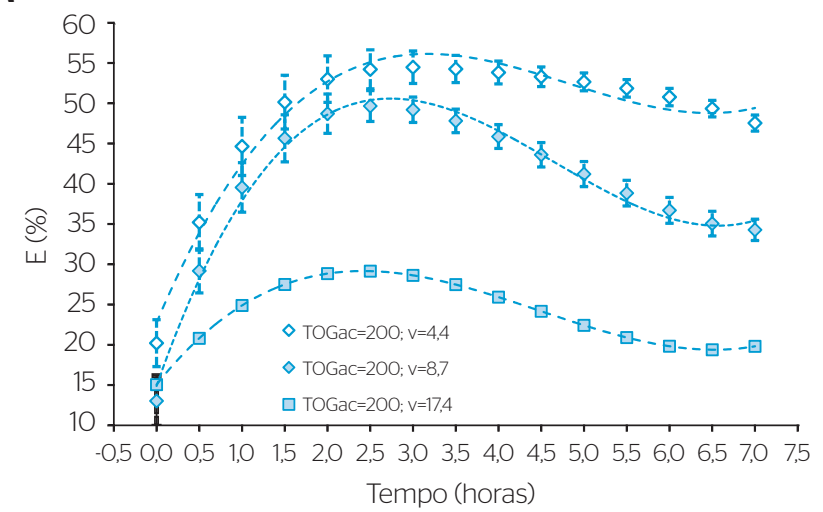

B

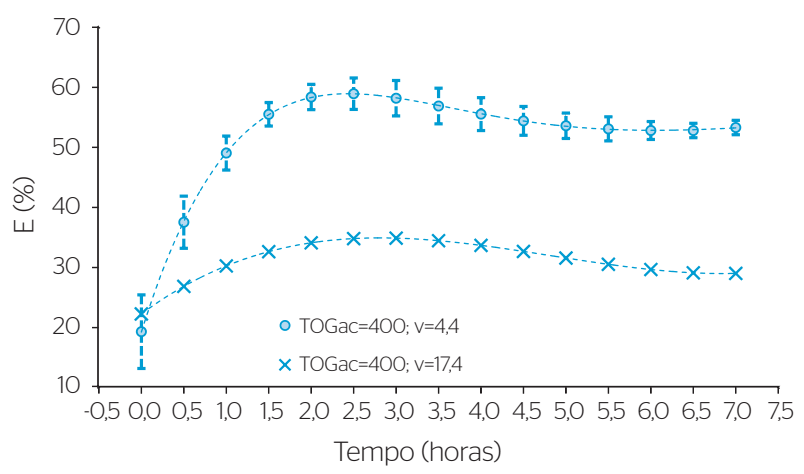

TOGac: teor de óleos e graxas na alimentação do coalescedor; v: velocidade do fluido; E: eficiência de tratamento.

Figura 4 - Eficiência de tratamento ao longo do tempo (A) para teor de óleos e graxas na alimentação do coalescedor de 200 mg. $\mathrm{L}^{-1} \mathrm{e}$ valores de velocidade superficial de 4,4; 8,7 e 17,4 m.h-1 e (B) para teor de óleos e graxas na alimentação do coalescedor de 400 mg. $\mathrm{L}^{-1}$ e valores de velocidade superficial de 4,4 e $17,4 \mathrm{~m} \cdot \mathrm{h}^{-1}$. 
foram similares nos dois trabalhos. Além disso, o diâmetro médio de gotas usados por esses autores foi de $20 \mu \mathrm{m}$, enquanto neste trabalho esse parâmetro foi de 3 a $8 \mu \mathrm{m}$.

Com relação ao diâmetro de gotas, um aspecto importante a ser destacado é que a literatura cita que o TOGac não tem efeito significativo na \%E, comportamento que contrasta com o que foi concluído por Sokolović, Sokolović e Doković (1997). Outros autores (SPIELMAN, 1968; SHERONY \& KINTNER, 1971) afirmaram que existem parâmetros muito mais relevantes que o TOGac, como, por exemplo, o diâmetro da gota de óleo.

\section{Influência da força de interação do leito}

A Figura 6 mostra a eficiência de remoção de óleo ao longo do tempo para duas curvas com os mesmos parâmetros testados (TOGac de $200 \mathrm{mg} . \mathrm{L}^{-1} \mathrm{e} \mathrm{v}$ de $4,4 \mathrm{~m} \cdot \mathrm{h}^{-1}$ ), mas com a resina que compunha o leito em condições de uso diferentes: uma curva representa a resina nova, e a outra, a resina usada, ou seja, a resina após a realização de vários ensaios anteriores.

A curva que representa a resina usada é a mesma já mostrada em figuras anteriores e, conforme citado, ela foi obtida a partir da realização de seis testes repetidos. Já a curva representativa da resina nova foi baseada na realização de apenas um teste.

Conforme pode-se observar, todas as curvas apresentam comportamentos semelhantes. Entretanto, a curva da resina nova resultou em uma eficiência muito maior, também apresentando inicialmente uma queda mais acentuada que as outras. A partir de seis horas de teste decorridas, essa queda já tende a apresentar uma estabilização.

Tais comportamentos podem ser indicativos de que a interação entre a resina e a fase oleosa leva à perda do desempenho da mesma, resultante da adsorção desse óleo na resina. Além disso, o processo de limpeza se mostrou incapaz de regenerar seu desempenho. Deve-se destacar que o procedimento de limpeza do leito visou apenas recuperar a ko, processo que foi realizado com sucesso.

Com relação ao diâmetro médio das gotas de óleo, as análises realizadas demostraram que não ocorrem alterações significativas entre os valores da alimentação e do coalescedor. As gotas de saída possuem diâmetros muito próximos ou, às vezes, levemente mais baixos do que as gotas da alimentação. Esse resultado é esperado, uma vez que as gotas de diâmetros maiores, resultantes do processo de coalescência que ocorre no interior do leito, devem ficar retidas na unidade se a configuração hidráulica do equipamento for projetada de forma adequada, como se supõe que seja o caso.

Portanto, várias considerações puderam ser estabelecidas sobre a utilização do coalescedor de leito como um processo de pré-tratamento de efluentes visando uma primeira remoção do óleo emulsionado. Uma delas foi que o coalescedor pode atuar de modo a remover uma carga inicial de óleo do afluente, contribuindo para reduzir a carga de óleo

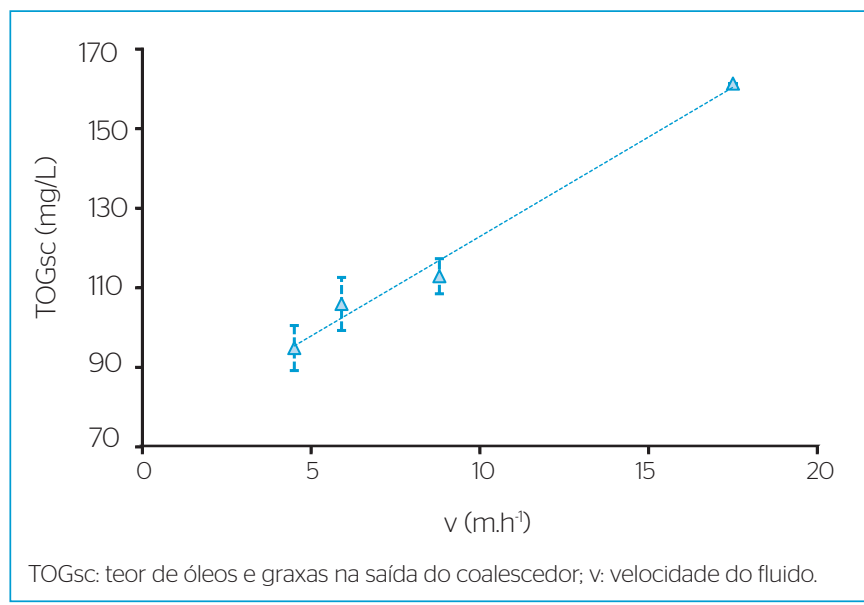

Figura 5 - Variação do teor de óleos e graxas na saída do coalescedor em função da velocidade para teor de óleos e graxas na alimentação do coalescedor de $200 \mathrm{mg}^{-\mathrm{L}^{-1}}$ decorridas 4 horas de teste.

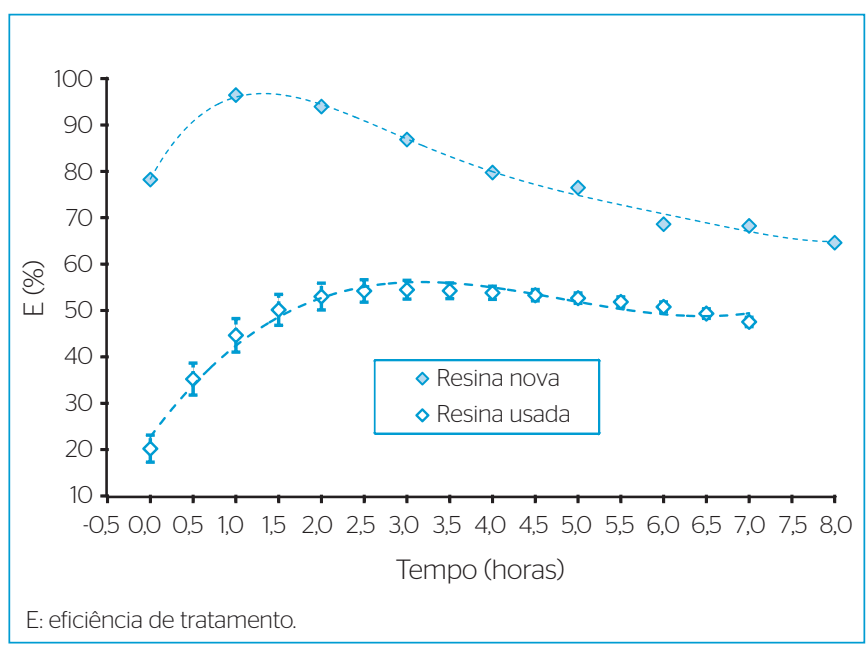

Figura 6 - Eficiência de tratamento ao longo do tempo para teor de óleos

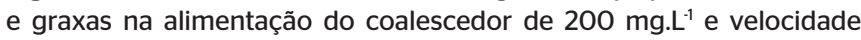
superficial de $4,4 \mathrm{~m} \cdot \mathrm{h}^{-1}$ para as resinas nova e usada.

a ser alimentada na unidade de remoção de óleo a ser usada a jusante. O coalescedor poderá operar com uma eficiência maior ou menor, em função da necessidade; para tanto, basta ajustar a v.

Outra consideração importante foi que o processo de tratamento a ser utilizado a jusante deve operar com uma carga de óleo mais elevada no período inicial de operação do coalescedor, quando a eficiência de remoção de óleo deste último é mais baixa do que a do período em que ele opera em estado estacionário. Para TOGam de $200 \mathrm{mg} . \mathrm{L}^{-1} \mathrm{e} \mathrm{v}$ de $4,4 \mathrm{~m} \cdot \mathrm{h}^{-1}$ esse período inicial pode ocorrer entre duas e quatro horas após a partida do processo (Figura 6). A estratégia a ser utilizada para superar esse obstáculo pode ser a saturação prévia do leito do coalescedor com a água oleosa a ser tratada, a fim de que o processo integrado já incie no estado estacionário. Na prática, essa condição poderia ser obtida por meio da recirculação do efluente oleoso a ser tratado pelo coalescedor. 


\section{CONCLUSÕES}

Os coalescedores de leito granular representam uma importante técnica de remoção de óleo de efluentes. Os resultados mostraram que o processo opera em estado estacionário decorridas 3-4 horas da partida da planta, mostrando ainda que os valores de TOGac testados influenciam na eficiência do processo para as velocidades testadas, enquanto a velocidade superficial influencia de modo inversamente proporcional, ou seja, a eficiência cai à medida que a velocidade aumenta para os valores de TOGac testados.

O processo já tem sido utilizado em instalações industriais com o objetivo de tratar o efluente, visando a obtenção de valores de eficiência mais elevados, a ponto de possibilitar o seu descarte em concordância com a legislação.

Neste estudo, o processo foi utilizado como um sistema de pré-tratamento para determinados processos de remoção de óleo, ficando demonstrado que pode apresentar resultados muito bons, além de várias vantagens adicionais. Entre essas vantagens estão a de possibilitar uma operação mais simplificada, uma redução significativa no volume de leito a ser utilizado e a de tratar emulsões $\mathrm{O} / \mathrm{A}$ com gotas de óleo de difícil remoção na faixa de micros ou submicros.

Assim, essa aplicação, como pré-tratamento, pode ser importante para uma primeira remoção de óleo de um efluente oleoso, permitindo que o processo de tratamento a ser utilizado a jusante opere de forma otimizada e com um tempo de campanha operacional mais longo, ou seja, sem a necessidade de interrupções para retrolavagem ou limpeza com tanta frequência.

Ressalta-se que a aplicação do coalescedor de leito como um processo de pré-tratamento pode ser um aspecto extremamente importante, quando se considera que determinados processos de tratamento poderiam ser viabilizados caso fossem utilizados a jusante do mesmo. Ocorre que alguns processos são mais complexos de serem operados para tratamento de efluentes oleosos, apesar de produzirem um efluente final com uma qualidade muito boa. O coalescedor de leito viabilizaria a aplicação desse processo, que seria usado apenas com o fim de promover o polimento final do efluente.

\section{AGRADECIMENTOS}

O desenvolvimento deste trabalho contou com o apoio e a colaboração do Laboratório de Membranas da Coppe - Instituto Alberto Luiz Coimbra de Pós-Graduação e Pesquisa de Engenharia, da Universidade Federal do Rio de Janeiro (UFRJ) e da Petrobras, por meio do Laboratório de Tratamento e Reuso de Águas (LARA), ligado ao Centro de Pesquisas e Desenvolvimento Leopoldo Américo Miguez de Mello (Cenpes/ Petrobras), e da Universidade Petrobras.

\section{REFERÊNCIAS}

AUSTIN, D.G. (1979) Coalescence of secundary dispersions. (Ph.D. Dissertation) - University of Aston in Birmingham, U.K.

GOVEDARICA, D.D.; SOKOLOVIĆ, R.M.S.; SOKOLOVIĆ, D.S.; SOKOLOVIĆ, S.M. (2013) A novel approach for the estimation of the efficiency of steady-state fiber bed coalescence. Separation and Purification Technology, v. 104, p. 268-275.

LI, J. \& GU, Y. (2005) Coalescence of oil-in-water emulsions in fibrous and granular beds. Separation And Purification Technology, v. 42, n. 1. p. 1-13.

SAREEN, S.S.; ROSE, P.M.; GUDESEN, R.C.; KINTNER, R.C. (1966) Coalescence in Fibrous Bed. A.I.Ch.E. Journal, v. 12, n. 6, p. 1045-1050.

SHERONY, D.F. \& KINTNER, R.C. (1971) Coalescence of an Emulsion in a Fibrous Bed: Part I - Theory. The Canadian Journal of Chemical Engineering, v. 49, p. 314-320.

SOKOLOVIĆ, R.M.S.; GOVEDARICA, D.D.; SOKOLOVIĆ, D.S. (2O10) Separation of oil-in-water emulsion using two coalescers of different geometry. Journal of Hazardous Materials, v. 175 , p. 1001-1006.
SOKOLOVIĆ, R.M.S.; VULIĆ, T.J.; SOKOLOVIĆ, S.M. (2007) Effect of bed length on steady-state coalescence of oil-in-water emulsion. Separation and purification technology, v. 56, p. 79-84.

SOKOLOVIĆ, R.M.S.; VULIĆ, T.J.; SOKOLOVIĆ, S.M.; NEDUCIN, R.P.M (2003) Effect of fibrous bed permeability on steady-state coalescence. Industrial \& Engineering Chemistry Research, v. 42, n. 13, p. 3098-3102.

SOKOLOVIĆ, R.M.S.; SOKOLOVIĆ, S.M.; DOKOVIĆ, B.D. (1997) Effect of working conditions on bed coalescence of an oil-in-water emulsion using a polyurethane foam bed. Industrial \& Engineering Chemistry Research, v. 36, n. 11, p. 4949-4953.

SPIELMAN, L.A. (1968) Separation of finely dispersed liquid-liquid suspensions by flow through fibrous media (Ph.D. Dissertation) University of California, Berkeley.

SPIELMAN, L.A. \& SU, Y.P. (1977) Coalescence of oil-in-water suspensions by flow through porous media, Industrial. Engineering \& Chemical Fundamentals, v. 16, n. 2, p. 272-282

THOMAS, J.E. (Org.) (2001) Fundamentos da engenharia de petróleo. 2 ed. Rio de Janeiro: Interciencia. 